\title{
USG Characteristics of Rare but Curable Thyroid Malignancy- Thyroid Lymphoma
}

\author{
Shivani Sidana, Shariq Rashid Masoodi, Moomin Hussain Bhat
}

Department of Endocrinology, SKIMS

Primary thyroid lymphomas (PTL) are rare tumors, accounting for less than $2 \%$ of all malignant thyroid tumors. PTL comprise of only $2.5 \%$ of all non-Hodgkin lymphomas. PTL occur mostly in the fifth to seventh decade, with the malefemale ratio being $1: 3$.

The classical presentation is women in the sixth decade with a history of chronic lymphocytic thyroiditis and rapidly growing firm diffuse thyroid mass.

Preexisting Hashimoto's thyroiditis is a well-recognized risk factor for the development of PTL. Reported incidence of Hashimoto's thyroiditis in patients with PTL is $30-100 \%$.

Therapeutic strategies of PTL is totally different from other thyroid malignancies, hence distinction of this tumor from other thyroid diseases/malignancies is important for proper clinical decision making. Accurate distinction of PTL from other thyroid conditions is made by histology, but there are certain image characteristics that help to predict the differentiation before histology.

Ota et al described the sonographic appearance of PTL in the largest series currently reported in the literature, and he classified PTL into nodular, diffuse, and mixed types based on the sonographic analysis.

Diffuse PTLs are markedly hypoechoic with heterogeneous internal echoes. Calcification in PTL is rare. However, micro calcification as well as macro calcification have been seen in diffuse PTL.

Sonographic characteristics of PTL are similar in

\begin{tabular}{|l|l|}
\multicolumn{2}{|c|}{ Access this article online } \\
\hline
\end{tabular}

appearance to diffuse Hashimoto's thyroiditis. There is no specific sonographic feature for differentiating diffuse PTL from Hashimoto thyroiditis; however, posterior acoustic enhancement has been reported to be useful in discriminating PTL from Hashimoto's thyroiditis.

Rapid growth (usually within the previous 1-3 months), painless thyroid enlargement, and pressure symptoms (mainly involving the aero digestive tract) are the common clinical presentations of PTL

Hence, combination of sonographic and clinical features may facilitate the diagnosis of diffuse PTL

We present a classical case of PTL, 70 year old female, with long standing hashimoto thyroiditis for last 5 years on $100 \mathrm{mcg}$ levothyroxine replacement, presented with painless neck swelling of predominately left side for last 2 months. Ultrasonography revealed enlarged left lobe of thyroid (58 x $48 \mathrm{~mm}$ ) showing hypoechoic nodularity and micro-calcifications, displacing left CCA and juglar vessels. Right lobe of thyroid showed hypoechoic lesion 12 x $8 \mathrm{~mm}$, with no calcification. FNAC from left lobe of thyroid revealed features of non-Hodgkins lymphoma. Patient was presently registered under RCC and further treatment was being planned.

\section{Correspondence:}

Dr. Shivani Sidana, M.D.

Department of Endocrinology, SKIMS

Email: shivani66sidana@gmail.com

How to cite this article: Sidana S, Masoodi SR, Bhat MH. USG Characteristics of rare but curable thyroid malignancy- Thyroid Lymphoma. jms [Internet]. 2019May27 [cited 2019May27];22(2). Available from: http://www.jmsskims.org/ index.php/jms/article/view/459

Received: 21-05-2019 Accepted: 27-05-2019 
Sidana S; et al USG Characteristics of Rare but Curable Thyroid Malignancy

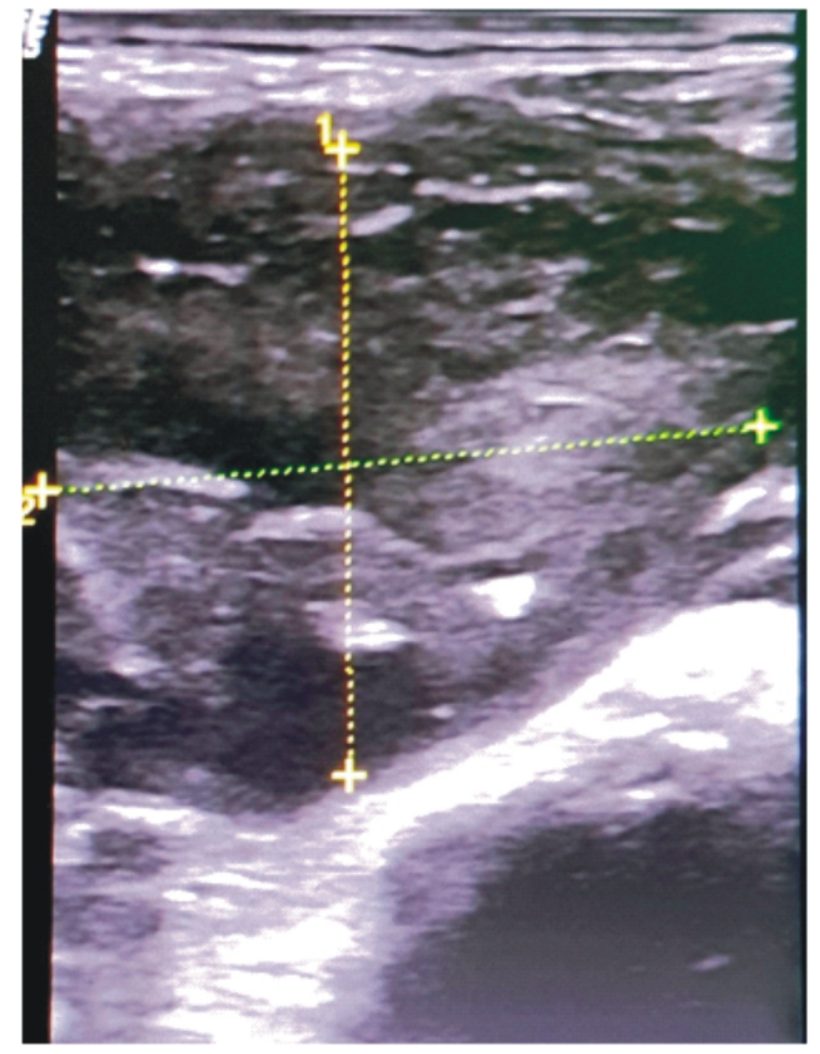

Fig 1: USG Left Thyroid lobe showing heterogeneous lesion with hypo and hyperechoic areas with microcalcificaltion

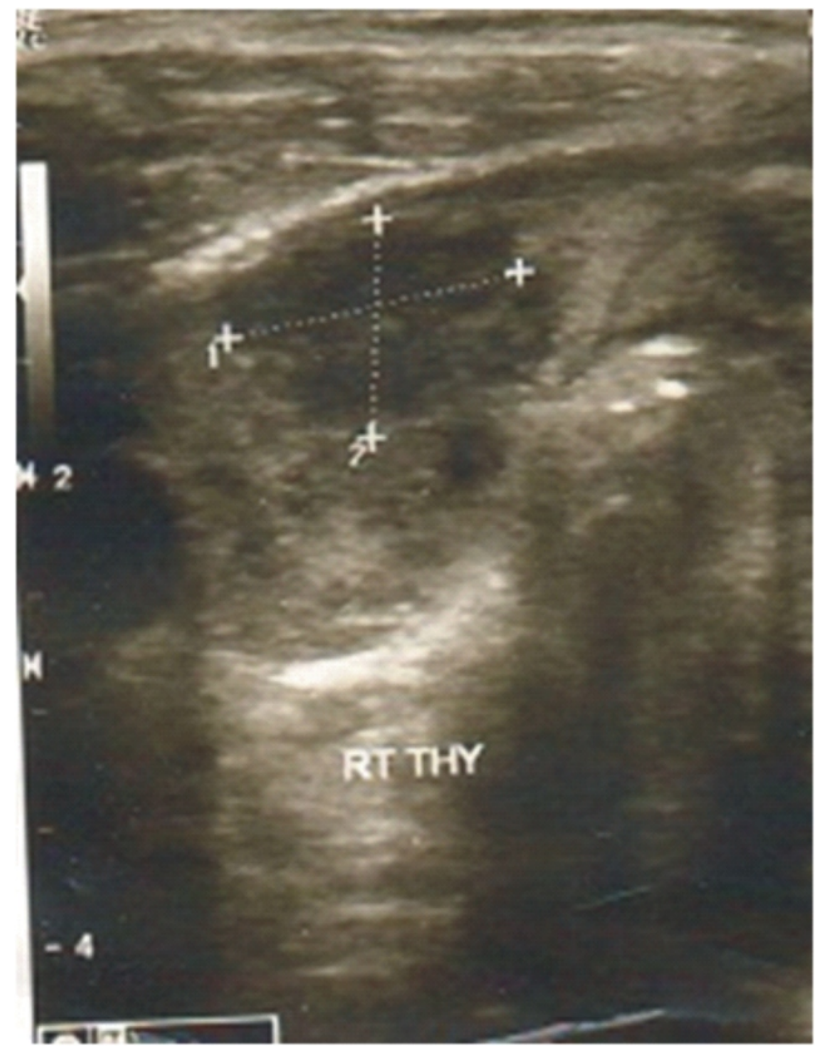

Fig 2: USG showing Right thyroid lobe with hypoechoic nodule 